\title{
Utility of neurophysiology in the diagnosis of tethered cord syndrome
}

\author{
Victoria Leung, BSc, ${ }^{1}$ Jeffery Pugh, MD,, ${ }^{1,2}$ and Jonathan A. Norton, PhD²,3 \\ ${ }^{1}$ Division of Neurosurgery, Department of Surgery, and ${ }^{2}$ Centre for Neuroscience, University of Alberta, Edmonton, Alberta; and \\ ${ }^{3}$ Division of Neurosurgery, Department of Surgery, University of Saskatchewan, Saskatoon, Saskatchewan, Canada
}

\begin{abstract}
OBJECT The diagnosis of tethered cord syndrome (TCS) remains difficult, and the decision to operate is even more complex. The objective of this study was to examine how detailed examination of neurophysiological test results can affect the diagnosis for patients undergoing a surgical cord release.
\end{abstract}

METHODS Patients undergoing tethered spinal cord releases were matched by age and sex with control patients undergoing scoliosis correction in the absence of spinal cord pathology. The latency and width of the P37 peak of the posterior tibial nerve somatosensory evoked potential (SSEP) and the motor evoked potential (MEP) latencies were examined. Immediate changes as a result of the surgical procedure were reported.

RESULTS The width of the P37 response differed significantly between TCS and control patients and changed significantly during the surgical procedure. Nonsignificant trends were seen in SSEP and MEP latencies.

CONCLUSIONS The width of the P37 response may be a useful marker for TCS and may play a role in presurgical decision making.

http://thejns.org/doi/abs/10.3171/2014.10.PEDS1434

KEY WORDS tethered cord syndrome; motor evoked potential; somatosensory evoked potential; diagnostics; spine; technique

$\mathrm{T}$ lethered cord syndrome (TCS) is a constellation of symptoms and signs that vary in severity and presentation. Pain and/or neurological, orthopedic, and urological deficits are believed to result from excessive tension on the spinal cord caused by tethering of the lower cord to inelastic tissue ${ }^{10}$ The causes of TCS also vary; one of the simplest causes is a fat-infiltrated and abnormally thick filum terminale. Normally, the viscoelasticity of the filum allows for slight adaptive movement of the conus medullaris, which buffers the distal spinal cord from the traction that occurs during flexion and extension of the spine. With fatty infiltration, however, the filum loses its viscoelasticity and instead tethers the distal end of the spinal cord to bone or other inelastic tissue. One hypothesis is that traction causes reduced blood supply to the cord, hypoxia, metabolic imbalances, and reduced excitability of the distal spinal cord and nerve roots. ${ }^{3,4,10}$ The cauda equina contains nerve roots responsible for sensory and motor innervation of the lower extremities, the urinary bladder, and urethral and anal sphincters, as well as sexual function. Thus, traction-induced impairment of these structures can cause sensorimotor complications. Common clinical findings include retention of urine and feces, incontinence, recurrent urinary tract infections, leg or foot length discrepancy, foot deformities, gait abnormalities, scoliosis, weakness in the legs, and sensory changes. ${ }^{1,8}$ TCS is typically diagnosed early in life, although symptoms can appear later, during the teenage years and adulthood. ${ }^{9}$ Unfortunately, many of these symptoms are difficult to recognize in children, particularly infants; for pediatric patients with minor forms of tethered spinal cord, there is debate regarding the best management strategies.

Clinical suspicion of a fatty filum is confirmed by MRI; a thickened (usually $>3 \mathrm{~mm}$ in diameter) filum is easily visible on T1-weighted MR images. ${ }^{8}$ A tethered spinal cord is confirmed by images that demonstrate elongation 
and caudal descent of the conus medullaris, ${ }^{8}$ in addition to clinical presentation. ${ }^{4}$ It is generally agreed that symptomatic patients with a low-lying conus (below the L-2 vertebral body) and a fatty filum should undergo surgical untethering. ${ }^{2,8}$ However, there is controversy around whether asymptomatic patients with a low conus medullaris, or even a normally positioned conus and a fatty filum, should undergo prophylactic surgery. ${ }^{2,8}$ Although untethering surgery can prevent the progression of symptoms in most patients and improve deficits in others, ${ }^{2,3}$ the risks must be weighed when the indication for surgery is less clear. Although some asymptomatic patients can remain asymptomatic, for others, delayed diagnosis and treatment can result in irreversible deficits that could have been prevented with prophylactic surgery. ${ }^{1,2,6-8}$ Untethering surgery carefully dissects lumbosacral nerve roots from their cause of tethering, but nearby neural elements supplying the lower extremities or sphincters are vulnerable to injury during dissection. Such damage would potentially cause or worsen the very problems the surgery is trying to prevent or improve. Therefore, to reduce the possibility of inadvertent injury to neural structures during TCS surgery, neurophysiological intraoperative monitoring is widely used. ${ }^{5}$

We believe that differences in clinical neurophysiological data between patients with a tethered spinal cord undergoing surgical release and otherwise healthy age- and sex-matched control patients undergoing surgical correction of scoliosis can reveal patterns that will aid in the diagnosis of a tethered spinal cord and predict the prognosis of untethering surgery. We predict that identifying such electrophysiological markers of the tethered spinal cord may thereby improve the ability to recognize those patients who will benefit from surgery.

\section{Methods}

We retrospectively reviewed medical records and electrophysiological data of patients who received treatment at the University of Alberta and University of Saskatchewan departments of surgery from January 2010 through May 2013. We compared neurophysiological potentials of 14 patients with a fatty filum terminale who had undergone surgical release of a tethered cord with those of neurologically normal age- and sex-matched control patients who had undergone surgical correction of idiopathic scoliosis.

At both institutions, a Cascade Elite system (Cadwell) was used to record somatosensory evoked potentials (SSEPs) and motor evoked potentials (MEPs). All recordings were analyzed off-line. SSEPs were obtained by using our standard protocol with posterior tibial nerve stimulation and recordings from the popliteal fossa, upper cervical spinal cord, and somatosensory cortex. In this study, we analyzed only the cortical responses, namely the P37 response. We chose this peak because it is often the clearest peak in our intraoperative recordings. We examined the duration of the P37 peak (i.e., the time from the initial deviation from the baseline to the zero crossing) (Fig. 1). We determined the initial deviation from the baseline as being more than 2 standard deviations from the baseline between 10 and $20 \mathrm{msec}$ after the stimulation. MEPs were elicited through electrodes placed in the scalp

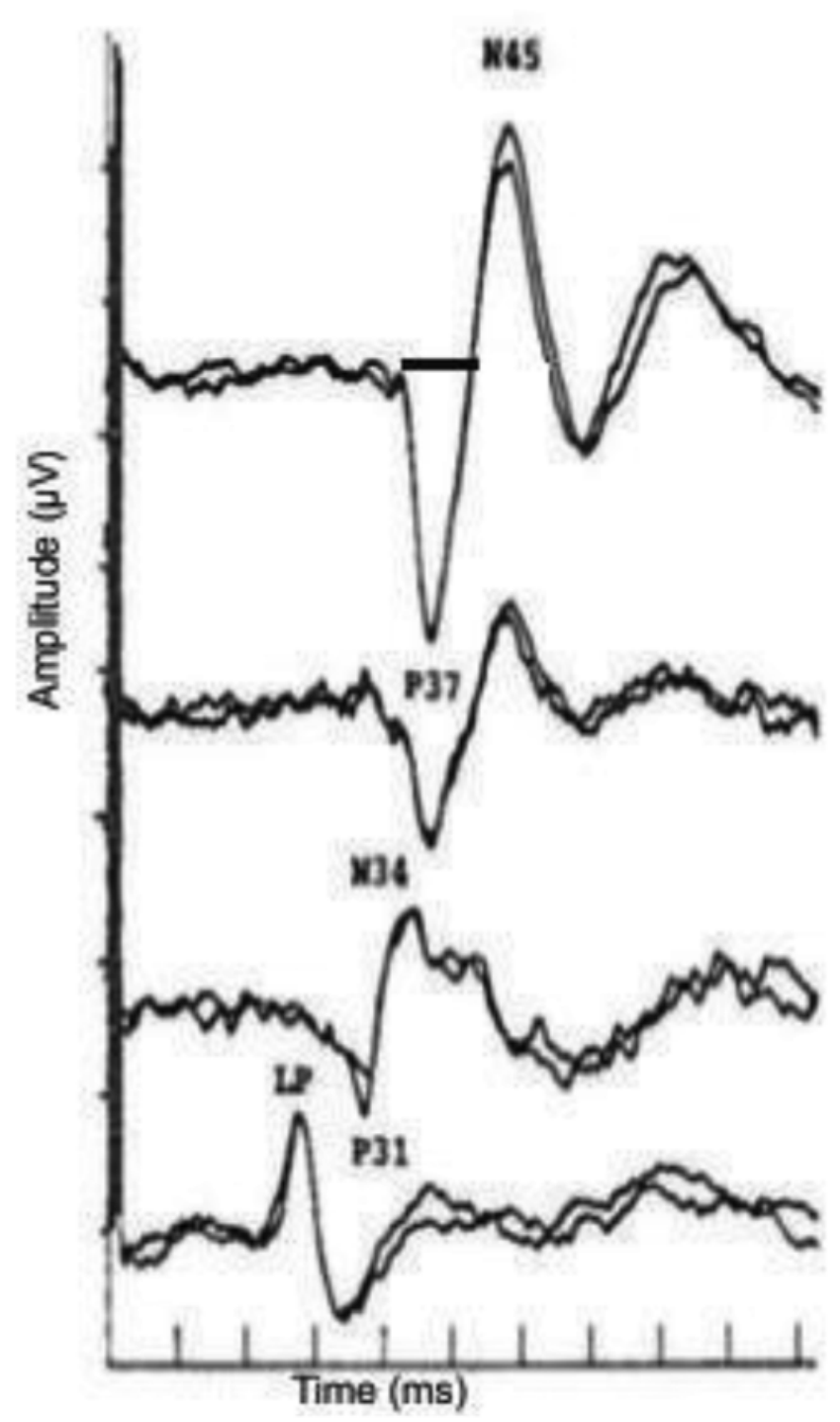

FIG. 1. An illustrative SSEP from posterior tibial nerve stimulation (200usec pulse width, $30 \mathrm{~mA}, 4.44 \mathrm{~Hz}$ ). The uppermost trace is from cortical electrodes and shows the P37 and N45 complex. The solid bar illustrates the measure we describe as the width of the P37 response.

overlying the motor cortices. Responses were recorded in segmentally innervated muscles in the lower limbs by using subdermal needles. We examined the responses from the tibialis anterior and adductor hallucis longus muscles (sphincter muscle potentials were not recorded for most control patients). We recorded the delay between the onset of the responses in the tibialis anterior and the adductor hallucis longus muscles, as outlined in Fig. 1.

Statistical analyses were performed by using MATLAB (MathWorks). Paired t-tests were used to compare data between TCS patients and their matched control patients. Unpaired t-tests were used to compare group data (TCS vs control populations).

\section{Results}

A total of 14 TSC patients and age- and size-matched controls were retrospectively entered into the study. All 
TCS patients had undergone surgery and were believed by the neurosurgeons to have had a symptomatic tethered cord. The surgeons' determination that patients are symptomatic surgical candidates may impose a bias on patient selection because only patients with the most severe neurological symptoms underwent surgery. For all patients we were able to record robust SSEPs and MEPs. Only studies in which the same number of pulses and interpulse intervals (MEPs) and pulse widths (SSEPs) had been recorded were selected for comparison. Among patients, the number of MEP pulses varied between 4 and 7; interpulse intervals varied between 1 and $2.4 \mathrm{msec}$. Stimulus pulse widths for the SSEPs were 200-450 $\mu$ sec.

The responses in the upper limbs and higher lumbar spinal levels seemed to be similar between the 2 groups. In the areas of interest, both sensory and motor potentials differed significantly. The delay between the onset of MEPs in the tibialis anterior and adductor hallucis muscles was greater among TCS patients than among control patients ( 2.7 vs $2.6 \mathrm{msec}$, respectively; $\mathrm{p}<0.005$, paired $\mathrm{t}$-test). However, this difference is small and probably falls within the measurement error of the system.

A greater difference was seen in the breadth of the P37 peak (17.2 vs $14.9 \mathrm{msec}(\mathrm{p}<0.005$, paired t-test ). This finding represents a $15 \%$ difference in the breadth of the P37 response between the groups. This statistical significance was retained when compared with our normative data $(17.2 \pm 0.65$ vs $14.8 \pm 1.3 \mathrm{msec}, \mathrm{p}<0.05$, t-test $)$. Of note, the breadth of the peak decreased significantly after detethering of the spinal cord $(17.2 \mathrm{msec}$ before release and $16.1 \mathrm{msec}$ after release, $\mathrm{p}<0.05$, paired t-test). Surgically released tethered cords demonstrated a reliable early correction in the breadth of the P37 peak with a shift toward normative data $(16.1 \pm 0.52$ vs $14.8 \pm 1.3 \mathrm{msec}, \mathrm{p}<$ 0.05 , t-test). Surgical correction of the scoliosis resulted in changes in the latency of the P37 peak but not the breadth of the peak. Our experienced anesthesia team kept the level of anesthesia very stable, and no neurophysiologic changes were noted during procedures other than during detethering (TCS patients) or spine derotation and other corrective procedures (control patients).

Figure 2 is a scatter plot of the width of the P37 peak for each pair of TCS and control patients. Table 1 illustrates the neurophysiological recording results for TCS patients.

\section{Case Discussion}

We compared neurophysiological data from patients who underwent tethered cord release with data from patients who underwent scoliosis surgery. All TCS patients, therefore, were pre-identified as having a tethered spinal cord. In one subsequent case, the senior author (J.A.N.) was so concerned with the prolongation of the P37 peaks bilaterally at the onset of a scoliosis surgery that the procedure was halted and a subsequent MR image identified a tethered spinal cord. Detethering of this spinal cord during a second procedure resulted in a rapid return toward a more normal P37 peak (from $16.5 \mathrm{msec}$ to $14.2 \mathrm{msec}$ ). Three months later, the scoliosis surgery was performed with full monitoring, the P37 peaks remained unchanged at $14.2 \mathrm{msec}$, and the patient was asymptomatic. Follow-up

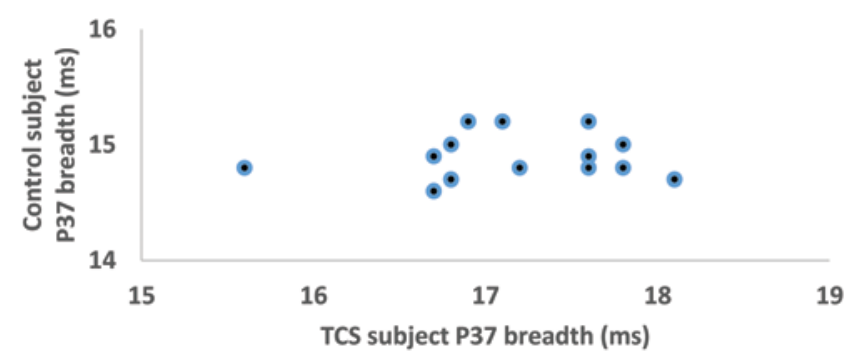

FIG. 2. A scatter plot of the widths of the P37 peak for TCS and control patients. Each TCS patient is paired with a control patient who is the same age and undergoing surgery for idiopathic scoliosis. Figure is available in color online only.

of this patient 1 year later indicated no change in the P37 width and continued absence of symptoms.

\section{Discussion}

TCS refers to a constellation of symptoms and signs related to lower spinal cord dysfunction. Surgical release of the tethered cord relieves the traction forces on the spinal cord and can prevent the progression of symptoms, improve or stabilize their severity, or prevent future deficits from developing. The present diagnostic gold standard is still clinical and radiological assessment. This study provides some preliminary evidence that neurophysiologic testing might also be able to provide evidence of a tethered spinal cord.

The breadth of the peak is not a normal measure in clinical neurophysiology; more typical markers are latencies and amplitudes. The P37 peak represents the arrival of the sensory volley in the primary sensory cortex. The distribution of the origin of the potential is on the medial aspect of the hemisphere; maximum positivity is closest to the vertex. The breadth may be a marker of demyelination; in patients with multiple sclerosis, it is often broader than usual. In TCS patients, there are no other specific markers of demyelination; indeed, the peripheral latencies are well within normal limits as are those in the upper limbs and

TABLE 1. Neurophysiological recording results

\begin{tabular}{cccc}
\hline & & \multicolumn{2}{c}{ SSEP Width (msec) } \\
\cline { 3 - 4 } Patient No. & MEP Delay (msec) & Preop & Postop \\
\hline 1 & 2.43 & 17.8 & 15.9 \\
\hline 2 & 2.36 & 17.6 & 16.3 \\
\hline 3 & 2.89 & 18.1 & 17.1 \\
\hline 4 & 2.32 & 17.6 & 16.1 \\
\hline 5 & 2.65 & 16.9 & 15.7 \\
\hline 6 & 2.94 & 17.8 & 16.4 \\
\hline 7 & 3.05 & 17.6 & 16.7 \\
\hline 8 & 2.87 & 16.7 & 16.3 \\
\hline 9 & 3.22 & 15.6 & 14.9 \\
\hline 10 & 2.97 & 16.7 & 16.1 \\
\hline 11 & 2.15 & 16.8 & 16.0 \\
\hline 12 & 2.68 & 17.2 & 16.3 \\
\hline 13 & 2.39 & 17.1 & 16.5 \\
\hline 14 & 3.01 & 16.8 & 15.7 \\
\hline
\end{tabular}


lumbar-innervated muscles and dermatomes (JA Norton, personal observations). The breadth of the peak therefore seems to indicate that there may be some focal slowing, presumably at the filum terminale. There was a small immediate reduction in the breadth of the peak after release of the tethered cord, although a broader peak did remain. We do not have follow-up data for any of these patients.

Most surgical procedures to de-tether spinal cords are performed in younger children, and we did not have data on healthy control patients with which to pair them. This is undoubtedly a limitation of our study. Performing SSEP studies in nonsedated young children is difficult, but combining the studies with MR images from sedated children is feasible, and indeed we have done so on many occasions. The breadth of the SSEP peak for TCS patients differed statistically from that for the control patients, but MEP measures did not differ statistically from those of our control population and so are of limited diagnostic utility.

\section{Conclusions}

Neurophysiological testing may be a useful adjunct in the diagnosis of TCS, especially for asymptomatic or clinically stable patients. More work is required to extend the study to include younger children. Further study will examine the association between neurophysiological and clinical changes, the time course of such changes (including during detethering), and presence or absence of a radiological correlation.

\section{References}

1. Agarwalla PK, Dunn IF, Scott RM, Smith ER: Tethered cord syndrome. Neurosurg Clin N Am 18:531-547, 2007

2. Bao N, Chen ZH, Gu S, Chen QM, Jin HM, Shi CR: Tight filum terminale syndrome in children: analysis based on positioning of the conus and absence or presence of lumbosacral lipoma. Childs Nerv Syst 23:1129-1134, 2007
3. Bui CJ, Tubbs RS, Oakes WJ: Tethered cord syndrome in children: a review. Neurosurg Focus 23(2):E2, 2007

4. Filippidis AS, Kalani MY, Theodore N, Rekate HL: Spinal cord traction, vascular compromise, hypoxia, and metabolic derangements in the pathophysiology of tethered cord syndrome. Neurosurg Focus 29(1):E9, 2010

5. Galloway GM, Zamel K: Neurophysiologic intraoperative monitoring in pediatrics. Pediatr Neurol 44:161-170, 2011

6. Pugh JA, Tubbs RS, Oakes WJ: Lipomyelomeningocele/tethered cord: prophylactic untethering, in Jallo GI, Kothbauer KF, Pradilla G (eds): Controversies in Pediatric Neurosurgery. New York: Thieme, 2010, pp 204-207

7. Satar N, Bauer SB, Shefner J, Kelly MD, Darbey MM: The effects of delayed diagnosis and treatment in patients with an occult spinal dysraphism. J Urol 154:754-758, 1995

8. Tubbs RS, Pugh J, Wellons JC III: Tethered spinal cord: fatty filum terminale, meningocele manqué, and dermal sinus tracts, in Winn HR (ed): Youmans Neurological Surgery. Philadelphia: Elsevier Sauders, 2011, Vol 2, pp 2227-2232

9. Yamada S, Lonser RR, Colohan ART, Yamada CT: Tethered cord syndrome in adult and late-teenage patients without neural spinal dysraphism, in Yamada S (ed): Tethered Cord Syndrome in Children and Adults, ed 2. New York: Thieme, 2010, pp 162-179

10. Yamada S, Won DJ, Yamada SM: Pathophysiology of tethered cord syndrome: correlation with symptomatology. Neurosurg Focus 16(2):E6, 2004

\section{Author Contributions}

Conception and design: Norton, Pugh. Acquisition of data: Norton. Analysis and interpretation of data: Norton, Leung. Drafting the article: Norton, Leung. Critically revising the article: Norton, Pugh. Reviewed submitted version of manuscript: Norton. Approved the final version of the manuscript on behalf of all authors: Norton. Statistical analysis: Norton. Study supervision: Pugh.

\section{Correspondence}

Jonathan Norton, Division of Neurosurgery, Department of Surgery, Royal University Hospital, 103 Hospital Dr., Saskatoon, SK S7N 0W8, Canada. email: j.norton@usask.ca. 